\section{sciendo}

DOI: $10.2478 /$ awutm-2018-0017
Analele Universităţii de Vest,

Timişoara

Seria Matematică - Informatică

LVI, 2, (2018), 81- 103

\title{
On uniform exponential trisplitting for cocycles of linear operators in Banach spaces
}

Larisa Elena Biriş, Claudia Luminiţa Mihiţ, Traian Ceauşu, and Ioan-Lucian Popa

Dedicated to Professor Mihail Megan on the occasion of his 70th birthday

\begin{abstract}
The aim of this paper is to study the concept of uniform exponential trisplitting for skew-product semiflow in Banach spaces. This concept is a generalisation of the well-known concept of uniform exponential trichotomy. We obtain necessary and sufficient conditions for this concept of Datko's type. a characterisation in terms of Lyapunov functions is provided. The results are obtained from the point of view of the projector families, i.e. invariant and strongly invariant.
\end{abstract}

AMS Subject Classification (2000). 34D05, 34D09

Keywords. Cocycle over a semiflow, invariant projector, strongly invariant projector, uniform exponential trisplitting

\section{Introduction}

The problem of skew-product dynamical systems has been investigated extensively in the literature. For the readers' convenience we refer to the books of C. Pötzsche [14], P.E. Kloeden and M. Rasmusen [9] and their references. Over the past few years, the theory of linear skew-product semiflows has been improved and used in the study of asymptotic behaviors of nonautonomous systems, by reducing the behavior of the system to the behavior of its solution, which is modelled by a linear skew-product semiflow. This notion arises 
naturally when one considers the linearization along the invariant manifold of a dynamical system.

The dichotomy concept plays an important role in the qualitative theory of dynamical systems. Notable contributions in this direction have been reported. Starting with R. J. Sacker and G. R. Sell in [20], [21], this concept was also studied by C. Chicone and Y. Latushkin [7], N. T. Huy [10], S. N. Chow and H. Leiva [8], Y. Latushkin, S. Montgomery-Smith and T. Raldolph [11], Y. Latushkin and R. Schnaubelt [12], A.L. Sasu and B. Sasu [22].

In some situations, particularly in the nonautonomous setting, the concept of uniform exponential dichotomy is too restrictive and it is important to consider more general behaviors. From this point of view, two different directions have been considered: first dealing with the so called nonuniform dichotomies that depend on the initial times, and second one that considers growth rates which do not imply an exponential dichotomy behavior, the so called splitting concepts.

Regarding the first approach we can point out the works of L. Barreira and C. Valls [2], L. Biriş and M. Megan [5], M. Megan, B. Sasu and A.L. Sasu [15], M. Megan, C. Stoica and L. Buliga [17] and M. Megan and C. Stoica [18]. An extension of this concept leads us to trichotomy concepts and we can mention the works of L. Biriş and R. Retezan [6] and M. Megan, C. Stoica and L. Buliga [16].

Regarding the second approach we can mention the paper of B. Aulbach and J. Kalkbrenner [1] where this concept has been introduced for difference equations. Some results concerning the above concept can be found in L. E. Biriş, T. Ceauşu and C. L. Mihiţ [3], M. Megan and I-L. Popa [13] for difference equations and in [19] for noninvertible evolution operators.

In this paper we will make an attempt to extend the splitting concept in a more general setting. We consider two concepts of exponential trisplitting for cocycles of linear operators. These concepts use two ideas of projections: invariant and strongly invariant for the respective cocycle. We note that the study of exponential trisplitting for discrete skew-product semiflows is presented in [4].

Let us outline the structure of the paper. In the next section we briefly recall the notions of cocycle over a semiflow which will be used later. We characterize the concept of exponential trisplitting with invariant projections in Theorem 2.1. and Theorem 2.2. and a counterexample showing that this concept is different from the classical concept of uniform exponential trichotomy is presented in Example 2.1. The last section deals with the concept of exponential trisplitting with strongly invariant projections, the characterizations are presented in Proposition 3.3 and Proposition 3.4. 


\section{Cocycles over semiflows}

Let us denote by $X$ a metric space, by $V$ a Banach space and by $\mathcal{B}(V)$ the Banach algebra of all bounded linear operators on $V$. The norm on $V$ and on $\mathcal{B}(V)$ will be denoted by $\|\cdot\|$. Let $I$ be the identity operator on $V$ and we also shall denote by $\mathbb{R}_{+}$the set of nonnegative real numbers and $Y=X \times V$.

Definition 2.1. A mapping $S: \mathbb{R}_{+} \times X \rightarrow X$ is called a semiflow on $X$, if:

$\left(s_{1}\right) S(0, x)=x$, for every $x \in X$;

$\left(s_{2}\right) S\left(t_{1}, S\left(t_{2}, x\right)\right)=S\left(t_{1}+t_{2}, x\right)$, for all $\left(t_{1}, t_{2}, x\right) \in \mathbb{R}_{+}^{2} \times X$.

Definition 2.2. A mapping $C: \mathbb{R}_{+} \times X \rightarrow \mathcal{B}(V)$ is called a cocycle over the semiflow $S: \mathbb{R}_{+} \times X \rightarrow X$ on the space $Y=X \times V$ if

$\left(c_{1}\right) C(0, x) v=v$, for every $(x, v) \in Y$;

$\left(c_{2}\right) C\left(t_{1}, S\left(t_{2}, x\right)\right) C\left(t_{2}, x\right)=C\left(t_{1}+t_{2}, x\right)$, for all $\left(t_{1}, t_{2}, x\right) \in \mathbb{R}_{+}^{2} \times X$.

Moreover, if

$\left(c_{3}\right)$ there are $M \geq 1$ and $\omega>0$ such that

$$
\|C(t, x) v\| \leq M e^{\omega t}\|v\|, \text { for all }(t, x, v) \in \mathbb{R}_{+} \times Y,
$$

then we say that $C$ has an exponential growth.

The linear skew-product semiflow associated with the above cocycle is the dynamical system $\pi=(S, C)$ on $Y=X \times V$, defined by

$$
\pi: \mathbb{R}_{+} \times Y \rightarrow Y, \pi(t, x, v)=(S(t, x), C(t, x) v)
$$

Definition 2.3. A mapping $P: X \rightarrow \mathcal{B}(V)$ is called a family of projectors on $X$ if

$$
P^{2}(x)=P(x), \quad \text { for all } x \in X .
$$

Definition 2.4. A family of projectors $P: X \rightarrow \mathcal{B}(V)$ is said to be invariant for the cocycle $C$ if

$$
C(t, x) P(x)=P(S(t, x)) C(t, x), \quad \text { for all }(t, x) \in \mathbb{R}_{+} \times X .
$$

Definition 2.5. A family of three projectors $\mathcal{P}=\left\{P_{1}, P_{2}, P_{3}\right\}$ is called orthogonally if $P_{1}(x)+P_{2}(x)+P_{3}(x)=I$ and $P_{k}(x) P_{j}(x)=0$, for all $x \in X$ and all $k, j \in\{1,2,3\}$ with $k \neq j$. 
Definition 2.6. The orthogonal family $\mathcal{P}=\left\{P_{1}, P_{2}, P_{3}\right\}$ is invariant for $C$ if $P_{j}$ is invariant for $C$, for all $j \in\{1,2,3\}$. In this case we denote by $(C, \mathcal{P})$.

Definition 2.7. The pair $(C, \mathcal{P})$ admits uniform exponential trisplitting if there are $N \geq 1$ and $\alpha, \beta, \gamma, \delta \in \mathbb{R}$ with $\alpha<\beta$ and $\gamma<\delta$ such that

$\left(\right.$ uet $\left._{1}\right)\left\|C(t, x) P_{1}(x) v\right\| \leq N e^{\alpha t}\left\|P_{1}(x) v\right\|$

$\left(\right.$ uet $\left._{2}\right) e^{\beta t}\left\|P_{2}(x) v\right\| \leq N\left\|C(t, x) P_{2}(x) v\right\|$

$\left(\right.$ uet $\left._{3}\right) e^{\gamma t}\left\|C(t, x) P_{3}(x) v\right\| \leq N\left\|P_{3}(x) v\right\|$

$\left(\right.$ uet $\left._{4}\right)\left\|P_{3}(x) v\right\| \leq N e^{\delta t}\left\|C(t, x) P_{3}(x) v\right\|$, for all $(t, x, v) \in \mathbb{R}_{+} \times Y$.

Remark 2.1. If $\alpha<0<\beta$ and $\gamma<0<\delta$, then we say that the pair $(C, \mathcal{P})$ admits uniform exponential trichotomy.

Remark 2.2. If $P_{3}(x)=0$, for all $x \in X$, then we say that the pair $(C, \mathcal{P})$ admits uniform exponential splitting.

Remark 2.3. If $P_{3}(x)=0$, for all $x \in X$ and $\alpha<0<\beta$, then we say that the pair $(C, \mathcal{P})$ admits uniform exponential dichotomy.

Remark 2.4. If the pair $(C, \mathcal{P})$ has uniform exponential trichotomy, then the pair $(C, \mathcal{P})$ admits uniform exponential trisplitting. The converse affirmation is not generally true.

Example 2.1. We consider $\mathcal{C}$ the metric space of all continuous functions $f: \mathbb{R}_{+} \rightarrow \mathbb{R}_{+}$, with the topology of uniform convergence on compact subsets of $\mathbb{R}_{+}$. Let $f \in \mathcal{C}$ be a decreasing function with $l=\lim _{t \rightarrow+\infty} f(t)>0$. Let $s \in \mathbb{R}_{+}$and $f_{s}: \mathbb{R}_{+} \rightarrow \mathbb{R}_{+}$be defined by $f_{s}(t)=f(s+t)$. Then $X=$ $\left\{\overline{f_{s} \in \mathcal{C}: s \in \mathbb{R}_{+}}\right\}$is a metric space. The mapping $S: \mathbb{R}_{+} \times X \rightarrow X, S(t, x)=$ $x_{t}$ is a semiflow on $X$. We consider $V=\mathbb{R}^{3}$ with the norm $\left\|\left(v_{1}, v_{2}, v_{3}\right)\right\|=$ $\left|v_{1}\right|+\left|v_{2}\right|+\left|v_{3}\right|$. It is easy to prove that $C: \mathbb{R}_{+} \times X \rightarrow \mathcal{B}(V)$, defined by $C(t, x) v=\left(e^{g(t)} v_{1}, e^{h(t)} v_{2}, e^{g(t)} v_{3}\right)$, where $g(t)=\pi x(0) t+\int_{0}^{t} x(s) d s, h(t)=$ $2 \pi x(0) t-\int_{0}^{t} x(s) d s$ is a cocycle over the semiflow $S$. We consider the family of projectors $P_{1}, P_{2}, P_{3}: X \rightarrow \mathcal{B}(V)$ given by

$$
P_{1}(x) v=\left(v_{1}, 0,0\right), P_{2}(x) v=\left(0, v_{2}, 0\right), P_{3}(x) v=\left(0,0, v_{3}\right) .
$$


Then, $P_{j}$ are invariant for $C$, for all $j=1,2,3$. We have that

$$
\begin{aligned}
& \left\|C(t, x) P_{1}(x) v\right\|=e^{g(t)}\left|v_{1}\right|=e^{g(t)}\left\|P_{1}(x) v\right\|, \\
& \left\|C(t, x) P_{2}(x) v\right\|=e^{h(t)}\left|v_{2}\right|=e^{h(t)}\left\|P_{2}(x) v\right\|, \\
& \left\|C(t, x) P_{3}(x) v\right\|=e^{g(t)}\left|v_{3}\right|=e^{g(t)}\left\|P_{3}(x) v\right\|,
\end{aligned}
$$

for all $(t, x, v) \in \mathbb{R}_{+} \times Y$. Because $g(t) \leq \pi x(0) t+t x(0)=(\pi+1) x(0) t$ and $h(t) \geq 2 \pi x(0) t-t x(0)=(2 \pi-1) x(0) t$, it follows that the relations $\left(\right.$ uet $\left._{1}\right)$ and $\left(\right.$ uet $\left._{2}\right)$ hold for $N=1$ and $\alpha=(\pi+1) x(0)<(2 \pi-1) x(0)=\beta$.

Next, we observe that

$$
g(t)-2 \pi x(0) t \leq \pi x(0) t+t x(0)-2 \pi x(0) t=(1-\pi) x(0) t
$$

and

$$
g(t)-\pi x(0) t=\int_{0}^{t} x(s) d s>l t .
$$

It follows that the relations $\left(\right.$ uet $\left._{3}\right)$ and $\left(\right.$ uet $\left._{4}\right)$ hold for $N=1$ and $\gamma=$ $-2 \pi x(0)<-\pi x(0)=\delta$. So the pair $(C, \mathcal{P})$ admits uniform exponential trisplitting. The pair $(C, \mathcal{P})$ is not uniformly exponentially trichotomic. If we assume that $(C, \mathcal{P})$ is uniformly exponentially trichotomic, it follows that there exist $N \geq 1$ and $\alpha<0$ such that $e^{g(t)} \leq N e^{\alpha t}$, for all $t \in \mathbb{R}_{+}$. For $t \rightarrow+\infty$ we obtain a contradiction.

Proposition 2.1. The pair $(C, \mathcal{P})$ has uniform exponential trisplitting if and only if there exist $N \geq 1$ and $\alpha, \beta, \gamma, \delta \in \mathbb{R}$ with $\alpha<\beta$ and $\gamma<\delta$ with

$\left(u e t_{1}^{\prime}\right)\left\|C(t+s, x) P_{1}(x) v\right\| \leq N e^{\alpha t}\left\|C(s, x) P_{1}(x) v\right\|$

$\left(\right.$ uet $\left._{2}^{\prime}\right) e^{\beta t}\left\|C(s, x) P_{2}(x) v\right\| \leq N\left\|C(t+s, x) P_{2}(x) v\right\|$

$\left.\left(u_{e}^{\prime}\right)_{3}\right) e^{\gamma t}\left\|C(t+s, x) P_{3}(x) v\right\| \leq N\left\|C(s, x) P_{3}(x) v\right\|$

$\left(u e t_{4}^{\prime}\right)\left\|C(s, x) P_{3}(x) v\right\| \leq N e^{\delta t}\left\|C(t+s, x) P_{3}(x) v\right\|$,

for all $(t, s) \in \mathbb{R}_{+}$and all $(x, v) \in Y$.

Proof. Necessity. It follows from Definition 2.7 for $x \rightarrow S(s, x)$ and $v \rightarrow$ $C(s, x) v$.

Sufficiency. It is immediate for $s=0$.

Definition 2.8. The pair $(C, \mathcal{P})$ admits uniform exponential growth if there exist $N \geq 1$ and $\theta, \xi \in(0,+\infty)$ such that 
$\left(u e g_{1}\right)\left\|C(t, x) P_{1}(x) v\right\| \leq N e^{\theta t}\left\|P_{1}(x) v\right\|$

$\left(u e g_{2}\right)\left\|P_{2}(x) v\right\| \leq N e^{\theta t}\left\|C(t, x) P_{2}(x) v\right\|$

$\left(u e g_{3}\right)\left\|C(t, x) P_{3}(x) v\right\| \leq N e^{\xi t}\left\|P_{3}(x) v\right\|$

$\left(u e g_{4}\right)\left\|P_{3}(x) v\right\| \leq N e^{\xi t}\left\|C(t, x) P_{3}(x) v\right\|$, for all $(t, x, v) \in \mathbb{R}_{+} \times Y$.

Proposition 2.2. The pair $(C, \mathcal{P})$ has uniform exponential growth if and only if there exist $N \geq 1$ and $\theta, \xi \in(0,+\infty)$ such that

$\left(u e g_{1}^{\prime}\right)\left\|C(t+s, x) P_{1}(x) v\right\| \leq N e^{\theta t}\left\|C(s, x) P_{1}(x) v\right\|$

$\left(u e g_{2}^{\prime}\right)\left\|C(s, x) P_{2}(x) v\right\| \leq N e^{\theta t}\left\|C(t+s, x) P_{2}(x) v\right\|$

$\left(u e g_{3}^{\prime}\right)\left\|C(t+s, x) P_{3}(x) v\right\| \leq N e^{\xi t}\left\|C(s, x) P_{3}(x) v\right\|$

$\left(u e g_{4}^{\prime}\right)\left\|C(s, x) P_{3}(x) v\right\| \leq N e^{\xi t}\left\|C(t+s, x) P_{3}(x) v\right\|$, for all $(t, s) \in \mathbb{R}_{+}$and all $(x, v) \in Y$.

Proof. Necessity. It yields from Definition 2.8 for $x \rightarrow S(s, x)$ and $v \rightarrow$ $C(s, x) v$.

Sufficiency. It is immediate for $s=0$.

Remark 2.5. If the pair $(C, \mathcal{P})$ admits uniform exponential trisplitting then it admits uniform exponential growth.

Indeed, if $\alpha, \beta, \gamma, \delta \in \mathbb{R}$ with $\alpha<\beta$ and $\gamma<\delta$ are the constants of trisplitting then $\theta=\max \{\alpha,-\beta\}$ and $\xi=\max \{\delta,-\gamma\}$ are the constants of uniform exponential growth.

Definition 2.9. A cocycle $C: \mathbb{R}_{+} \times X \rightarrow \mathcal{B}(V)$ is called strongly measurable if for every $(x, v) \in Y$, the mapping $t \rightarrow C(t, x) v$ is measurable.

Definition 2.10. Let $C: \mathbb{R}_{+} \times X \rightarrow \mathcal{B}(V)$ be a strongly measurable cocycle. The pair $(C, \mathcal{P})$ admits uniform exponential trisplitting of Datko's type if there are $D \geq 1$ and $\mu, \nu, \omega, \eta \in \mathbb{R}$ with $\mu<\nu$ and $\omega<\eta$ such that for all $(t, x, v) \in \mathbb{R}_{+} \times Y$

$\left(u e d_{1}\right) \int_{t}^{\infty} e^{\mu(t-\tau)}\left\|C(\tau, x) P_{1}(x) v\right\| d \tau \leq D\left\|C(t, x) P_{1}(x) v\right\|$

$\left(u e d_{2}\right) \int_{0}^{t} e^{\nu(t-\tau)}\left\|C(\tau, x) P_{2}(x) v\right\| d \tau \leq D\left\|C(t, x) P_{2}(x) v\right\|$ 


$$
\begin{gathered}
\left(u e d_{3}\right) \int_{t}^{\infty} e^{\omega(\tau-t)}\left\|C(\tau, x) P_{3}(x) v\right\| d \tau \leq D\left\|C(t, x) P_{3}(x) v\right\| \\
\left(\text { ued }_{4}\right) \int_{0}^{t} e^{\eta(\tau-t)}\left\|C(\tau, x) P_{3}(x) v\right\| d \tau \leq D\left\|C(t, x) P_{3}(x) v\right\| .
\end{gathered}
$$

Theorem 2.1. Let $C: \mathbb{R}_{+} \times X \rightarrow \mathcal{B}(V)$ be a strongly measurable cocycle. The pair $(C, \mathcal{P})$ admits uniform exponential trisplitting if and only if $(C, \mathcal{P})$ has uniform exponential growth and admits uniform exponential trisplitting of Datko's type.

Proof. Necessity. We consider $N \geq 1$ and $\alpha, \beta, \gamma, \delta \in \mathbb{R}$ with $\alpha<\beta$ and $\gamma<\delta$ the constant in relation to which $(C, \mathcal{P})$ admits uniform exponential trisplitting. Then $N \geq 1, \theta=\max \{\alpha,-\beta\}$ and $\xi=\max \{\delta,-\gamma\}$ are the constants in relation to which $(C, \mathcal{P})$ admits uniform exponential growth. Let $\mu, \nu, \omega, \eta \in \mathbb{R}$ be such that $\alpha<\mu<\nu<\beta, \omega<\gamma<\delta<\eta$ and $(t, x, v) \in \mathbb{R}_{+} \times Y$. For $\left(\right.$ uet $\left._{1}^{\prime}\right) \Rightarrow\left(\right.$ ued $\left._{1}\right)$, we observe that

$$
\begin{aligned}
& \int_{t}^{\infty} e^{\mu(t-\tau)}\left\|C(\tau, x) P_{1}(x) v\right\| d \tau \leq N \int_{t}^{\infty} e^{\mu(t-\tau)} e^{\alpha(\tau-t)}\left\|C(t, x) P_{1}(x) v\right\| d \tau= \\
& =\frac{N}{\mu-\alpha}\left\|C(t, x) P_{1}(x) v\right\| .
\end{aligned}
$$

For $\left(u e t_{2}^{\prime}\right) \Rightarrow\left(u_{e d}\right)$, we observe that

$$
\begin{aligned}
& \int_{0}^{t} e^{\nu(t-\tau)}\left\|C(\tau, x) P_{2}(x) v\right\| d \tau \leq N \int_{0}^{t} e^{\nu(t-\tau)} e^{-\beta(t-\tau)}\left\|C(t, x) P_{2}(x) v\right\| d \tau= \\
& =\frac{N}{\beta-\nu}\left\|C(t, x) P_{2}(x) v\right\| .
\end{aligned}
$$

We shall prove $\left(u e t_{3}^{\prime}\right) \Rightarrow\left(\right.$ ued $\left._{3}\right)$.

$$
\begin{aligned}
& \int_{t}^{\infty} e^{\omega(\tau-t)}\left\|C(\tau, x) P_{3}(x) v\right\| d \tau \leq N \int_{t}^{\infty} e^{\omega(\tau-t)} e^{-\gamma(\tau-t)}\left\|C(t, x) P_{3}(x) v\right\| d \tau= \\
& =\frac{N}{\gamma-\omega}\left\|C(t, x) P_{3}(x) v\right\| .
\end{aligned}
$$


Finally, for $\left(u_{e}^{\prime} t_{4}\right) \Rightarrow\left(u_{e} d_{4}\right)$, we observe that

$$
\begin{aligned}
& \int_{0}^{t} e^{\eta(\tau-t)}\left\|C(\tau, x) P_{3}(x) v\right\| d \tau \leq N \int_{0}^{t} e^{\eta(\tau-t)} e^{\delta(t-\tau)}\left\|C(t, x) P_{3}(x) v\right\| d \tau= \\
& =\frac{N}{\eta-\delta}\left\|C(t, x) P_{3}(x) v\right\| .
\end{aligned}
$$

Sufficiency. Let $N \geq 1$ and $\theta, \xi \in(0,+\infty)$ be the constants in relation to which the pair $(C, \mathcal{P})$ has uniform exponential growth and $D \geq 1$ and $\mu, \nu, \omega, \eta \in \mathbb{R}$ with $\mu<\nu$ and $\omega<\eta$ the constants in relation to which the pair $(C, \mathcal{P})$ admits uniform exponential trisplitting of Datko type. We consider $(x, v) \in Y$. We use the conditions $\left(u e g_{1}^{\prime}\right)$ and $\left(u e d_{1}\right)$ to prove $\left(\right.$ uet $\left._{1}\right)$. We observe that if we suppose $t \geq 1$, then

$$
\begin{gathered}
e^{-\mu t}\left\|C(t, x) P_{1}(x) v\right\|=\int_{t-1}^{t} e^{-\mu t}\left\|C(t, x) P_{1}(x) v\right\| d \tau \leq \\
\leq N \int_{t-1}^{t} e^{-\mu t} e^{\theta(t-\tau)}\left\|C(\tau, x) P_{1}(x) v\right\| d \tau= \\
=N \int_{t-1}^{t} e^{(\theta-\mu)(t-\tau)} e^{-\mu \tau}\left\|C(\tau, x) P_{1}(x) v\right\| d \tau \leq \\
\leq N e^{|\theta-\mu|} \int_{t-1}^{t} e^{-\mu \tau}\left\|C(\tau, x) P_{1}(x) v\right\| d \tau \leq \\
\leq N e^{|\theta-\mu|} \int_{0}^{+\infty} e^{-\mu \tau}\left\|C(\tau, x) P_{1}(x) v\right\| d \tau \leq N e^{|\theta-\mu|} \cdot D \cdot\left\|P_{1}(x) v\right\| .
\end{gathered}
$$

If $t \in[0,1)$, then from $\left(u e g_{1}\right)$, it follows that

$$
\begin{aligned}
\left\|C(t, x) P_{1}(x) v\right\| & \leq N e^{\theta t}\left\|P_{1}(x) v\right\|=N e^{(\theta-\mu) t} e^{\mu t}\left\|P_{1}(x) v\right\| \leq \\
& \leq N e^{|\theta-\mu|} D e^{\mu t}\left\|P_{1}(x) v\right\| .
\end{aligned}
$$

We use the conditions $\left(u e g_{2}\right)$ and $\left(u e d_{2}\right)$ to prove $\left(\right.$ uet $\left._{2}\right)$. We observe that if we suppose $t \geq 1$, then

$$
e^{\nu t}\left\|P_{2}(x) v\right\|=\int_{0}^{1} e^{\nu t}\left\|P_{2}(x) v\right\| d \tau \leq N \int_{0}^{1} e^{\nu t} e^{\theta \tau}\left\|C(\tau, x) P_{2}(x) v\right\| d \tau=
$$




$$
\begin{gathered}
=N \int_{0}^{1} e^{(\theta+\nu) \tau} e^{\nu(t-\tau)}\left\|C(\tau, x) P_{2}(x) v\right\| d \tau \leq \\
\leq N e^{|\theta+\nu|} \int_{0}^{1} e^{\nu(t-\tau)}\left\|C(\tau, x) P_{2}(x) v\right\| d \tau \leq \\
\leq N e^{|\theta+\nu|} \int_{0}^{t} e^{\nu(t-\tau)}\left\|C(\tau, x) P_{2}(x) v\right\| d \tau \leq N e^{|\theta+\nu|} \cdot D \cdot\left\|C(t, x) P_{2}(x) v\right\| .
\end{gathered}
$$

If $t \in[0,1)$, then from $\left(u e g_{2}\right)$ it follows that

$$
e^{\nu t}\left\|P_{2}(x) v\right\| \leq e^{\nu t} N e^{\theta t}\left\|C(t, x) P_{2}(x) v\right\| \leq N \cdot D \cdot e^{|\theta+\nu|}\left\|C(t, x) P_{2}(x) v\right\| .
$$

We use the conditions $\left(u e g_{3}^{\prime}\right)$ and $\left(u e d_{3}\right)$ to prove $\left(u e t_{3}\right)$. Considering $t \geq 1$, we obtain

$$
\begin{gathered}
\left\|C(t, x) P_{3}(x) v\right\|=\int_{t-1}^{t}\left\|C(t, x) P_{3}(x) v\right\| d \tau \leq \\
\leq N \int_{t-1}^{t} e^{\xi(t-\tau)}\left\|C(\tau, x) P_{3}(x) v\right\| d \tau= \\
=N e^{-\omega t} \int_{t-1}^{t} e^{(\xi+\omega)(t-\tau)} e^{\omega \tau}\left\|C(\tau, x) P_{3}(x) v\right\| d \tau \leq \\
\leq N e^{-\omega t} e^{|\xi+\omega|} \int_{t-1}^{t} e^{\omega \tau}\left\|C(\tau, x) P_{3}(x) v\right\| d \tau \leq \\
\leq N e^{-\omega t} e^{|\xi+\omega|} \int_{0}^{+\infty} e^{\omega \tau}\left\|C(\tau, x) P_{3}(x) v\right\| d \tau \leq N e^{-\omega t} e^{|\xi+\omega|} \cdot D \cdot\left\|P_{3}(x) v\right\| .
\end{gathered}
$$

If $t \in[0,1)$, then from $\left(u e g_{3}\right)$ it follows that

$$
\begin{aligned}
\left\|C(t, x) P_{3}(x) v\right\| \leq N e^{\xi t}\left\|P_{3}(x) v\right\|=N e^{(\xi+\omega) t} e^{-\omega t}\left\|P_{3}(x) v\right\| \leq \\
\leq N e^{|\xi+\omega|} e^{-\omega t} D\left\|P_{3}(x) v\right\| .
\end{aligned}
$$


We use the conditions $\left(u e g_{4}\right)$ and $\left(u e d_{4}\right)$ to prove $\left(u e t_{4}\right)$. Let $t \geq 1$. Then,

$$
\begin{gathered}
\left\|P_{3}(x) v\right\|=\int_{0}^{1}\left\|P_{3}(x) v\right\| d \tau \leq N \int_{0}^{1} e^{\xi t}\left\|C(\tau, x) P_{3}(x) v\right\| d \tau= \\
=N e^{\eta t} \int_{0}^{1} e^{(\xi-\eta) \tau} e^{\eta(\tau-t)}\left\|C(\tau, x) P_{3}(x) v\right\| d \tau \leq \\
\leq N e^{\eta t} e^{|\xi-\eta|} \int_{0}^{1} e^{\eta(\tau-t)}\left\|C(\tau, x) P_{3}(x) v\right\| d \tau \leq \\
\leq N e^{\eta t} e^{|\xi-\eta|} \int_{0}^{t} e^{\eta(\tau-t)}\left\|C(\tau, x) P_{3}(x) v\right\| d \tau \leq N e^{\eta t} e^{|\xi-\eta|} \cdot D \cdot\left\|C(t, x) P_{3}(x) v\right\| .
\end{gathered}
$$

If $t \in[0,1)$, then from $\left(u e g_{4}\right)$ it follows that

$$
\begin{aligned}
& \left\|P_{3}(x) v\right\| \leq N e^{\xi t}\left\|C(t, x) P_{3}(x) v\right\|=N e^{(\xi-\eta) t} e^{\eta t}\left\|C(t, x) P_{3}(x) v\right\| \leq \\
& \leq N e^{|\xi-\eta|} D e^{\eta t}\left\|C(t, x) P_{3}(x) v\right\| .
\end{aligned}
$$

Definition 2.11. Let $C: \mathbb{R}_{+} \times X \rightarrow \mathcal{B}(V)$ be a strongly measurable cocycle. We say that $L=\left(L_{1}, L_{2}, L_{3}\right): \mathbb{R}_{+} \times Y \rightarrow \mathbb{R}_{+}^{3}$ is a Lyapunov function for the pair $(C, \mathcal{P})$ if there exist $a, b, c, d \in \mathbb{R}, a<b, c<d$ such that for all $(t, x, v) \in \mathbb{R}_{+} \times Y$

$\left(l_{1}\right) L_{1}\left(t, x, P_{1}(x) v\right)+\int_{0}^{t} e^{-a \tau}\left\|C(\tau, x) P_{1}(x) v\right\| d \tau \leq L_{1}\left(0, x, P_{1}(x) v\right)$

$\left(l_{2}\right) L_{1}\left(0, x, P_{2}(x) v\right)+\int_{0}^{t} e^{b(t-\tau)}\left\|C(\tau, x) P_{2}(x) v\right\| d \tau \leq L_{1}\left(t, x, P_{2}(x) v\right)$

$\left(l_{3}\right) L_{2}\left(t, x, P_{3}(x) v\right)+\int_{0}^{t} e^{c \tau}\left\|C(\tau, x) P_{3}(x) v\right\| d \tau \leq L_{2}\left(0, x, P_{3}(x) v\right)$

$\left(l_{4}\right) L_{3}\left(0, x, P_{3}(x) v\right)+\int_{0}^{t} e^{d(\tau-t)}\left\|C(\tau, x) P_{3}(x) v\right\| d \tau \leq L_{3}\left(t, x, P_{3}(x) v\right)$. 
Theorem 2.2. Let $C: \mathbb{R}_{+} \times X \rightarrow \mathcal{B}(V)$ be a strongly measurable cocycle. The pair $(C, \mathcal{P})$ has uniform exponential trisplitting if and only if $(C, \mathcal{P})$ has uniform exponential growth and there exists a Lyapunov function $L=$ $\left(L_{1}, L_{2}, L_{3}\right): \mathbb{R}_{+} \times Y \rightarrow \mathbb{R}_{+}^{3}$ for $(C, \mathcal{P})$ and a constant $M \geq 1$ such that for all $(t, x, v) \in \mathbb{R}_{+} \times Y$, the following relations are satisfied:

$\left(l_{1}^{\prime}\right) L_{1}\left(0, x, P_{1}(x) v\right) \leq M\left\|P_{1}(x) v\right\|$

$\left(l_{2}^{\prime}\right) L_{1}\left(t, x, P_{2}(x) v\right) \leq M\left\|C(t, x) P_{2}(x) v\right\|$

$\left(l_{3}^{\prime}\right) L_{2}\left(0, x, P_{3}(x) v\right) \leq M\left\|P_{3}(x) v\right\|$

$\left(l_{4}^{\prime}\right) L_{3}\left(t, x, P_{3}(x) v\right) \leq M\left\|C(t, x) P_{3}(x) v\right\|$

Proof. Necessity. We consider $N \geq 1, \alpha, \beta, \gamma, \delta \in \mathbb{R}$ with $\alpha<\beta$ and $\gamma<\delta$ and $\theta, \xi \in(0,+\infty)$ given by Definition 2.7 and Remark 2.5. Let $\mu, \nu, \omega, \eta \in \mathbb{R}$ be with $\mu<\nu$ and $\omega<\eta$ given in Theorem 2.1. Let $(t, x, v) \in \mathbb{R}_{+} \times Y$. We define $L=\left(L_{1}, L_{2}, L_{3}\right): \mathbb{R}_{+} \times Y \rightarrow \mathbb{R}_{+}^{3}$ given by

$$
\begin{gathered}
L_{1}(t, x, v)=\int_{t}^{+\infty} e^{-\mu \tau}\left\|C(\tau, x) P_{1}(x) v\right\| d \tau+\int_{0}^{t} e^{\nu(t-\tau)}\left\|C(\tau, x) P_{2}(x) v\right\| d \tau, \\
L_{2}(t, x, v)=\int_{t}^{+\infty} e^{\omega \tau}\left\|C(\tau, x) P_{3}(x) v\right\| d \tau \\
L_{3}(t, x, v)=\int_{0}^{t} e^{\eta(\tau-t)}\left\|C(\tau, x) P_{3}(x) v\right\| d \tau .
\end{gathered}
$$

First, we shall prove that $L$ is a Lyapunov function for the pair $(C, \mathcal{P})$.

$$
\begin{gathered}
\left(l_{1}\right) \quad L_{1}\left(0, x, P_{1}(x) v\right)=\int_{0}^{+\infty} e^{-\mu \tau}\left\|C(\tau, x) P_{1}(x) v\right\| d \tau= \\
=L_{1}\left(t, x, P_{1}(x) v\right)+\int_{0}^{t} e^{-\mu \tau}\left\|C(\tau, x) P_{1}(x) v\right\| d \tau ; \\
\left(l_{2}\right) \quad L_{1}\left(0, x, P_{2}(x) v\right)+\int_{0}^{t} e^{\nu(t-\tau)}\left\|C(\tau, x) P_{2}(x) v\right\| d \tau=
\end{gathered}
$$




$$
\begin{gathered}
=\int_{0}^{t} e^{\nu(t-\tau)}\left\|C(\tau, x) P_{2}(x) v\right\| d \tau=L_{1}\left(t, x, P_{2}(x) v\right) ; \\
\left(l_{3}\right) \quad L_{2}\left(t, x, P_{3}(x) v\right)+\int_{0}^{t} e^{\omega \tau}\left\|C(\tau, x) P_{3}(x) v\right\|= \\
=\int_{t}^{+\infty} e^{\omega \tau}\left\|C(\tau, x) P_{3}(x) v\right\| d \tau+\int_{0}^{t} e^{\omega \tau}\left\|C(\tau, x) P_{3}(x) v\right\| d \tau= \\
=\int_{0}^{+\infty} e^{\omega \tau}\left\|C(\tau, x) P_{3}(x) v\right\| d \tau=L_{2}\left(0, x, P_{3}(x) v\right) ;
\end{gathered}
$$

$\left(l_{4}\right) \quad L_{3}\left(0, x, P_{3}(x) v\right)+\int_{0}^{t} e^{\eta(\tau-t)}\left\|C(\tau, x) P_{3}(x) v\right\| d \tau=L_{3}\left(t, x, P_{3}(x) v\right)$.

Now, using Theorem 2.1, we observe that

$$
\begin{gathered}
L_{1}\left(t, x, P_{1}(x) v\right)+\int_{0}^{t} e^{-\mu \tau}\left\|C(\tau, x) P_{1}(x) v\right\| d \tau= \\
=\int_{0}^{+\infty} e^{-\mu \tau}\left\|C(\tau, x) P_{1}(x) v\right\| d \tau \leq D\left\|P_{1}(x) v\right\| ; \\
L_{1}\left(t, x, P_{2}(x) v\right)=\int_{0}^{t} e^{\nu(t-\tau)}\left\|C(\tau, x) P_{2}(x) v\right\| d \tau \leq D\left\|C(t, x) P_{2}(x) v\right\| ; \\
L_{2}\left(0, x, P_{3}(x) v\right)=\int_{0}^{+\infty} e^{\omega \tau}\left\|C(\tau, x) P_{3}(x) v\right\| d \tau \leq D\left\|P_{3}(x) v\right\| ; \\
L_{3}\left(t, x, P_{3}(x) v\right)=\int_{0}^{t} e^{\eta(\tau-t)}\left\|C(\tau, x) P_{3}(x) v\right\| d \tau \leq D\left\|C(t, x) P_{3}(x) v\right\| .
\end{gathered}
$$

We deduce that the conditions $\left(l_{1}^{\prime}\right),\left(l_{2}^{\prime}\right),\left(l_{3}^{\prime}\right)$ and $\left(l_{4}^{\prime}\right)$ hold.

Sufficiency. Using $\left(l_{1}\right),\left(u e g_{1}^{\prime}\right)$ and $\left(l_{1}^{\prime}\right)$, we shall prove $\left(\right.$ uet $\left._{1}\right)$. Let $(x, v) \in Y$. For $t \geq 1$, we observe that

$$
e^{-a t}\left\|C(t, x) P_{1}(x) v\right\|=\int_{t-1}^{t} e^{-a t}\left\|C(t, x) P_{1}(x) v\right\| d \tau \leq
$$




$$
\begin{gathered}
\leq N \int_{t-1}^{t} e^{\theta(t-\tau)} e^{-a t}\left\|C(\tau, x) P_{1}(x) v\right\| d \tau= \\
=N \int_{t-1}^{t} e^{-a \tau} e^{(\theta-a)(t-\tau)}\left\|C(\tau, x) P_{1}(x) v\right\| d \tau \leq \\
\leq N e^{|\theta-a|} \int_{0}^{t} e^{-a \tau}\left\|C(\tau, x) P_{1}(x) v\right\| \leq \\
\leq N e^{|\theta-a|} L_{1}\left(0, x, P_{1}(x) v\right) \leq N e^{|\theta-a|} M\left\|P_{1}(x) v\right\| .
\end{gathered}
$$

For $t \in[0,1)$, we have that

$$
\begin{aligned}
\left\|C(t, x) P_{1}(x) v\right\| & \leq N e^{\theta t}\left\|P_{1}(x) v\right\|=N e^{(\theta-a) t} e^{a t}\left\|P_{1}(x) v\right\| \leq \\
& \leq N e^{|\theta-a|} M e^{a t}\left\|P_{1}(x) v\right\| .
\end{aligned}
$$

Using $\left(l_{2}\right),\left(u e g_{2}\right)$ and $\left(l_{2}^{\prime}\right)$, we shall prove $\left(u e t_{2}\right)$. For $t \geq 1$, we have that

$$
\begin{gathered}
e^{b t}\left\|P_{2}(x) v\right\|=\int_{0}^{1} e^{b t}\left\|P_{2}(x) v\right\| d \tau \leq N \int_{0}^{1} e^{b t} e^{\theta \tau}\left\|C(\tau, x) P_{2}(x) v\right\| d \tau= \\
=N \int_{0}^{1} e^{b(t-\tau)} e^{(\theta+b) \tau}\left\|C(\tau, x) P_{2}(x) v\right\| d \tau \leq N e^{|\theta+b|} \int_{0}^{1} e^{b(t-\tau)}\left\|C(\tau, x) P_{2}(x) v\right\| d \tau \leq \\
\leq N e^{|\theta+b|} \int_{0}^{t} e^{b(t-\tau)}\left\|C(\tau, x) P_{2}(x) v\right\| d \tau \leq \\
\leq N e^{|\theta+b|} L_{1}\left(t, x, P_{2}(x) v\right) \leq N M e^{|\theta+b|}\left\|C(t, x) P_{2}(x) v\right\| .
\end{gathered}
$$

For $t \in[0,1)$ we observe that

$$
e^{b t}\left\|P_{2}(x) v\right\| \leq e^{b t} N e^{\theta t}\left\|C(t, x) P_{2}(x) v\right\| \leq N M e^{|\theta+b|}\left\|C(t, x) P_{2}(x) v\right\| .
$$

Using $\left(l_{3}\right),\left(u e g_{3}^{\prime}\right)$ and $\left(l_{3}^{\prime}\right)$, we shall prove $\left(\right.$ uet $\left._{3}\right)$. If $t \geq 1$, then

$$
\left\|C(t, x) P_{3}(x) v\right\|=\int_{t-1}^{t}\left\|C(t, x) P_{3}(x) v\right\| d \tau \leq
$$




$$
\begin{gathered}
\leq N \int_{t-1}^{t} e^{\xi(t-\tau)}\left\|C(\tau, x) P_{3}(x) v\right\| d \tau= \\
=N e^{-c t} \int_{t-1}^{t} e^{c \tau} e^{(c+\xi)(t-\tau)}\left\|C(\tau, x) P_{3}(x) v\right\| d \tau \leq \\
\leq N e^{-c t} e^{|c+\xi|} \int_{0}^{t} e^{c \tau}\left\|C(\tau, x) P_{3}(x) v\right\| d \tau \leq \\
\leq N e^{-c t} e^{|c+\xi|} L_{2}\left(0, x, P_{3}(x) v\right) \leq M N e^{-c t} e^{|c+\xi|}\left\|P_{3}(x) v\right\| .
\end{gathered}
$$

For $t \in[0,1)$, we deduce

$$
\begin{aligned}
\left\|C(t, x) P_{3}(x) v\right\| \leq & N e^{\xi t}\left\|P_{3}(x) v\right\|=N e^{(\xi+c) t} e^{-c t}\left\|P_{3}(x) v\right\| \leq \\
& \leq N M e^{|\xi+c|} e^{-c t}\left\|P_{3}(x) v\right\|
\end{aligned}
$$

Using $\left(l_{4}\right),\left(u e g_{4}\right)$ and $\left(l_{4}^{\prime}\right)$, we shall prove $\left(u e t_{4}\right)$. For $t \geq 1$, we have that

$$
\begin{gathered}
\left\|P_{3}(x) v\right\|=\int_{0}^{1}\left\|P_{3}(x) v\right\| d \tau \leq N \int_{0}^{1} e^{\xi \tau}\left\|C(\tau, x) P_{3}(x) v\right\| d \tau= \\
=N \int_{0}^{1} e^{d(\tau-t)} e^{(\xi-d) \tau} e^{d t}\left\|C(\tau, x) P_{3}(x) v\right\| d \tau \leq \\
\leq N e^{|\xi-d|} e^{d t} \int_{0}^{t} e^{d(\tau-t)}\left\|C(\tau, x) P_{3}(x) v\right\| d \tau \leq \\
\leq N e^{|\xi-d|} e^{d t} L_{3}\left(t, x, P_{3}(x) v\right) \leq N e^{|\xi-d|} e^{d t} M\left\|C(t, x) P_{3}(x) v\right\| .
\end{gathered}
$$

For $t \in[0,1)$, we observe that

$$
\begin{aligned}
& \left\|P_{3}(x) v\right\| \leq N e^{\xi t}\left\|C(t, x) P_{3}(x) v\right\| \leq N e^{(\xi-d) t} e^{d t}\left\|C(t, x) P_{3}(x) v\right\| \leq \\
& \leq N M e^{|\xi-d|} e^{d t}\left\|C(t, x) P_{3}(x) v\right\| .
\end{aligned}
$$




\section{Uniform exponential trisplitting with strongly in- variant projectors}

Let $\mathcal{P}=\left\{P_{1}, P_{2}, P_{3}\right\}$ be an orthogonal and invariant family of projectors for the cocycle $C: \mathbb{R}_{+} \times X \rightarrow \mathcal{B}(V)$ over the semiflow $S: \mathbb{R}_{+} \times X \rightarrow X$ on $Y=X \times V$.

Definition 3.1. We say that $\mathcal{P}=\left\{P_{1}, P_{2}, P_{3}\right\}$ is a strong invariant family of projections for the cocycle $C$ if for all $(t, x) \in \mathbb{R}_{+} \times X$ the map $C(t, x)$ is an isomorphism from Range $P_{j}(x)$ to Range $P_{j}(S(t, x)), j=2,3$.

Remark 3.1. If the family of projectors $\mathcal{P}=\left\{P_{1}, P_{2}, P_{3}\right\}$ is strong invariant for the cocycle $C: \mathbb{R}_{+} \times X \rightarrow \mathcal{B}(V)$ over the semiflow $S: \mathbb{R}_{+} \times X \rightarrow X$, then there exists $D_{j}: \mathbb{R}_{+} \times X \rightarrow \mathcal{B}(V)$ such that for all $(t, x) \in \mathbb{R}_{+} \times X$, $D_{j}(t, x)$ is an isomorphism from Range $P_{j}(S(t, x))$ to Range $P_{j}(x), j=2,3$ and

(i) $C(t, x) D_{j}(t, x) P_{j}(S(t, x))=P_{j}(S(t, x))$

(ii) $D_{j}(t, x) C(t, x) P_{j}(x)=P_{j}(x)=D_{j}(t, x) P_{j}(S(t, x)) C(t, x) P_{j}(x)$

(iii) $P_{j}(x) D_{j}(t, x) P_{j}(S(t, x))=D_{j}(t, x) P_{j}(S(t, x))$, for all $(t, x) \in \mathbb{R}_{+} \times X$.

Proposition 3.1. If $\mathcal{P}=\left\{P_{1}, P_{2}, P_{3}\right\}$ is strong invariant for the cocycle $C: \mathbb{R}_{+} \times X \rightarrow \mathcal{B}(V)$, then $D_{j}: \mathbb{R}_{+} \times X \rightarrow \mathcal{B}(V), j=2,3$ has the property:

(iv) $D_{j}(t+s, x) P_{j}(S(t+s, x))=D_{j}(s, x) D_{j}(t, S(s, x)) P_{j}(S(t+s, x))$,

for all $(t, s, x) \in \mathbb{R}_{+}^{2} \times X$.

Proof. For all $(t, s, x) \in \mathbb{R}_{+}^{2} \times X$, we have that

$$
\begin{aligned}
& D_{j}(t+s, x) P_{j}(S(t+s))=P_{j}(x) D_{j}(t+s, x) P_{j}(S(t+s, x))= \\
& =D_{j}(s, x) C(s, x) P_{j}(x) D_{j}(t+s, x) P_{j}(S(t+s, x))= \\
& =D_{j}(s, x) P_{j}(S(s, x)) C(s, x) D_{j}(t+s, x) P_{j}(S(t+s, x))= \\
& =D_{j}(s, x) D_{j}(t, S(s, x)) C(t, S(s, x)) P_{j}(S(s, x)) C(s, x) D_{j}(t+s, x)
\end{aligned}
$$

$P_{j}(S(t+s, x))=$

$=D_{j}(s, x) D_{j}(t, S(s, x)) C(t, S(s, x)) C(s, x) P_{j}(x) D(t+s, x) P_{j}(S(t+s, x))=$

$=D_{j}(s, x) D_{j}(t, S(s, x)) C(t+s, x) P_{j}(x) D_{j}(t+s, x) P_{j}(S(t+s, x))=$

$=D_{j}(s, x) D_{j}(t, S(s, x)) C(t+s, x) D_{j}(t+s, x) P_{j}(S(t+s, x))=$

$=D_{j}(s, x) D_{j}(t, S(s, x)) P_{j}(S(t+s, x))$. 
Proposition 3.2. The pair $(C, \mathcal{P})$ admits uniform a exponential growth if and only if there exist $N \geq 1$ and $\theta, \xi \in(0,+\infty)$ such that for all $(t, x, v) \in$ $\mathbb{R}_{+} \times Y$

$\left(u e g_{1}^{\prime \prime}\right)\left\|C(t, x) P_{1}(x) v\right\| \leq N e^{\theta t}\left\|P_{1}(x) v\right\|$

$\left(u e g_{2}^{\prime \prime}\right)\left\|D_{2}(t, x) P_{2}(S(t, x)) v\right\| \leq N e^{\theta t}\left\|P_{2}(S(t, x)) v\right\|$

$\left(u e g_{3}^{\prime \prime}\right)\left\|C(t, x) P_{3}(x) v\right\| \leq N e^{\xi t}\left\|P_{3}(x) v\right\|$

$\left(u e g_{4}^{\prime \prime}\right)\left\|D_{3}(t, x) P_{3}(S(t, x)) v\right\| \leq N e^{\xi t}\left\|P_{3}(S(t, x)) v\right\|$.

Proof. Let $(t, x, v) \in \mathbb{R}_{+} \times Y$. We shall prove that the condition $\left(u e g_{2}\right)$ from Definition 2.8 and $\left(u e g_{2}^{\prime \prime}\right)$ are equivalent. We have

$$
\begin{gathered}
\left\|D_{2}(t, x) P_{2}(S(t, x)) v\right\|=\left\|P_{2}(x) D_{2}(t, x) P_{2}(S(t, x)) v\right\| \leq \\
\leq N e^{\theta t}\left\|C(t, x) P_{2}(x) D_{2}(t, x) P_{2}(S(t, x)) v\right\|= \\
=N e^{\theta t}\left\|C(t, x) D_{2}(t, x) P_{2}(S(t, x)) v\right\|=N e^{\theta t}\left\|P_{2}(S(t, x)) v\right\| \quad \text { and } \\
\left\|P_{2}(x) v\right\|=\left\|D_{2}(t, x) P_{2}(S(t, x)) C(t, x) v\right\| \leq N e^{\theta t}\left\|P_{2}(S(t, x)) C(t, x) v\right\|= \\
=N e^{\theta t}\left\|C(t, x) P_{2}(x) v\right\| .
\end{gathered}
$$

Now, we shall prove the equivalence $\left(u e g_{4}\right) \Leftrightarrow\left(u e g_{4}^{\prime \prime}\right)$. We obtain

$$
\begin{gathered}
\left\|D_{3}(t, x) P_{3}(S(t, x)) v\right\|=\left\|P_{3}(x) D_{3}(t, x) P_{3}(S(t, x)) v\right\| \leq \\
\leq N e^{\xi t}\left\|C(t, x) P_{3}(x) D_{3}(t, x) P_{3}(S(t, x)) v\right\|=N e^{\xi t}\left\|P_{3}(S(t, x)) v\right\| \quad \text { and } \\
\left\|P_{3}(x) v\right\|=\left\|D_{3}(t, x) P_{3}(S(t, x)) C(t, x) v\right\| \leq \\
\leq N e^{\xi t}\left\|P_{3}(S(t, x)) C(t, x) v\right\|=N e^{\xi t}\left\|C(t, x) P_{3}(x) v\right\| .
\end{gathered}
$$

Proposition 3.3. Let $\mathcal{P}=\left\{P_{1}, P_{2}, P_{3}\right\}$ be a strong invariant family of projectors for the cocycle $C: \mathbb{R}_{+} \times X \rightarrow \mathcal{B}(V)$. Then the pair $(C, \mathcal{P})$ has uniform exponential trisplitting if and only if there exist $N \geq 1, \alpha, \beta, \gamma, \delta \in \mathbb{R}$ with $\alpha<\beta, \gamma<\delta$ such that for all $(t, x, v) \in \mathbb{R}_{+} \times Y$

$\left(u e t_{1}^{\prime \prime}\right)\left\|C(t, x) P_{1}(x) v\right\| \leq N e^{\alpha t}\left\|P_{1}(x) v\right\|$

$\left(u e t_{2}^{\prime \prime}\right) e^{\beta t}\left\|D_{2}(t, x) P_{2}(S(t, x)) v\right\| \leq N\left\|P_{2}(S(t, x)) v\right\|$

$\left(\right.$ uet $\left._{3}^{\prime \prime}\right) e^{\gamma t}\left\|C(t, x) P_{3}(x) v\right\| \leq N\left\|P_{3}(x) v\right\|$

$\left(u e t_{4}^{\prime \prime}\right)\left\|D_{3}(t, x) P_{3}(S(t, x)) v\right\| \leq N e^{\delta t}\left\|P_{3}(S(t, x)) v\right\|$, 
Proof. Let $(t, x, v) \in \mathbb{R}_{+} \times Y$. We prove the equivalence between $\left(\right.$ uet $\left._{2}^{\prime \prime}\right)$ and the condition $\left(\right.$ uet $\left._{2}\right)$ given by Definition 2.7. We have that

$$
\begin{aligned}
& e^{\beta t}\left\|P_{2}(x) v\right\|=e^{\beta t}\left\|D_{2}(t, x) C(t, x) P_{2}(x) v\right\|= \\
& =e^{\beta t}\left\|D_{2}(t, x) P_{2}(S(t, x)) C(t, x) v\right\| \leq N\left\|P_{2}(S(t, x)) C(t, x) v\right\|= \\
& =N\left\|C(t, x) P_{2}(x) v\right\| \text { and } \\
& e^{\beta t}\left\|D_{2}(t, x) P_{2}(S(t, x)) v\right\|=e^{\beta t}\left\|P_{2}(x) D_{2}(t, x) P_{2}(S(t, x)) v\right\| \leq \\
& \quad \leq N\left\|C(t, x) D_{2}(t, x) P_{2}(S(t, x)) v\right\|=N\left\|P_{2}(S(t, x)) v\right\| .
\end{aligned}
$$

Similarly, we obtain that the condition $\left(u_{e} t_{4}^{\prime \prime}\right)$ and the condition $\left(u_{4} t_{4}\right)$ from Definition 2.7 are equivalent. We have

$$
\begin{gathered}
\left\|P_{3}(x) v\right\|=\left\|D_{3}(t, x) C(t, x) P_{3}(x) v\right\|=\left\|D_{3}(t, x) P_{3}(S(t, x)) C(t, x) v\right\| \leq \\
\leq N e^{\delta t}\left\|P_{3}(S(t, x)) C(t, x) v\right\|=N e^{\delta t}\left\|C(t, x) P_{3}(x) v\right\|, \quad \text { respectively } \\
\left\|D_{3}(t, x) P_{3}(S(t, x)) v\right\|=\left\|P_{3}(x) D_{3}(t, x) P_{3}(S(t, x)) v\right\| \leq \\
\leq N e^{\delta t}\left\|C(t, x) D_{3}(t, x) P_{3}(S(t, x)) v\right\|=N e^{\delta t}\left\|P_{3}(S(t, x)) v\right\| .
\end{gathered}
$$

Proposition 3.4. Let $\mathcal{P}=\left\{P_{1}, P_{2}, P_{3}\right\}$ be a strong invariant family of projectors for the strongly measurable cocycle $C: \mathbb{R}_{+} \times X \rightarrow \mathcal{B}(V)$. Then the pair $(C, \mathcal{P})$ has uniform exponential trisplitting if and only if it has uniform exponential growth and there exist $D \geq 1$ and $\mu, \nu, \omega, \eta \in \mathbb{R}$ with $\mu<\nu$, $\omega<\eta$ such that for all $(t, x, v) \in \mathbb{R}_{+} \times Y$

$$
\begin{aligned}
& \left(u e d_{1}^{\prime}\right) \int_{t}^{+\infty} e^{\mu(t-\tau)}\left\|C(\tau, x) P_{1}(x) v\right\| d \tau \leq D\left\|C(t, x) P_{1}(x) v\right\| \\
& \left(u e d_{2}^{\prime}\right) \int_{0}^{t} e^{\nu(t-\tau)}\left\|D_{2}(t-\tau, S(\tau, x)) P_{2}(S(\tau, x)) v\right\| d \tau \leq D\left\|P_{2}(S(t, x)) v\right\| \\
& \left(\text { ued }_{3}^{\prime}\right) \int_{t}^{+\infty} e^{\omega(\tau-t)}\left\|C(\tau, x) P_{3}(x) v\right\| d \tau \leq D\left\|C(t, x) P_{3}(x) v\right\| \\
& \left(\text { ued }_{4}^{\prime}\right) \int_{0}^{t} e^{\eta(\tau-t)}\left\|D_{3}(t-\tau, S(\tau, x)) P_{3}(S(t, x)) v\right\| d \tau \leq D\left\|P_{3}(S(t, x)) v\right\| .
\end{aligned}
$$


Proof. Necessity. Let $(t, x, v) \in \mathbb{R}_{+} \times Y$. Using (uet $\left.t_{2}^{\prime \prime}\right)$ from Proposition 3.3 we obtain that

$$
e^{\beta(t-\tau)} \| D_{2}(t-\tau, S(\tau, x)) P_{2}\left(S(t-\tau, S(\tau, x)) v\|\leq N\| P_{2}(S(t-\tau, S(\tau, x)) v \|,\right.
$$

which is equivalent with

$$
e^{\beta(t-\tau)}\left\|D_{2}(t-\tau, S(\tau, x)) P_{2}(S(t, x)) v\right\| \leq N\left\|P_{2}(S(t, x)) v\right\| .
$$

We obtain that the condition (ued $d_{2}^{\prime}$ ) holds.

$$
\begin{gathered}
\int_{0}^{t} e^{\nu(t-\tau)}\left\|D_{2}(t-\tau, S(\tau, x)) P_{2}(S(t, x)) v\right\| d \tau \leq \\
\leq \int_{0}^{t} e^{\nu(t-\tau)} N e^{-\beta(t-\tau)}\left\|P_{2}(S(t, x)) v\right\| d \tau= \\
=N\left\|P_{2}(S(t, x)) v\right\| e^{(\nu-\beta) t} \int_{0}^{t} e^{(\beta-\nu) t} d \tau \leq \frac{N}{\beta-\nu}\left\|P_{2}(S(t, x)) v\right\| .
\end{gathered}
$$

Similarly, we obtain $\left(u e d_{4}^{\prime}\right)$. Using the condition $\left(u_{e}^{\prime \prime}\right)$ from Proposition 3.3, we have that

$$
\| D_{3}(t-\tau, S(\tau, x)) P_{3}\left(S(t-\tau, S(\tau, x)) v\left\|\leq N e^{\delta(t-\tau)}\right\| P_{3}(S(t-\tau, S(\tau, x)) v \|,\right.
$$

which is equivalent with

$$
\left\|D_{3}(t-\tau, S(\tau, x)) P_{3}(S(t, x)) v\right\| \leq N e^{\delta(t-\tau)}\left\|P_{3}(S(t, x)) v\right\| .
$$

It follows that the condition $\left(u e d_{4}^{\prime}\right)$ holds.

$$
\begin{aligned}
& \int_{0}^{t} e^{\eta(\tau-t)}\left\|D_{3}(t-\tau, S(\tau, x)) P_{3}(S(t, x)) v\right\| d \tau \leq \\
& \leq N\left\|P_{3}(S(t, x)) v\right\| \int_{0}^{t} e^{\eta(\tau-t)} e^{\delta(t-\tau)} d \tau \leq \frac{N}{\eta-\delta}\left\|P_{3}(S(t, x)) v\right\| .
\end{aligned}
$$

Sufficiency. Let $(x, v) \in Y$. From $\left(u e g_{1}^{\prime \prime}\right)$ and $\left(u e d_{1}^{\prime}\right)$ we obtain that

$$
\left\|C(t, x) P_{1}(x) v\right\| \leq N D e^{|\theta-\mu|} e^{\mu t}\left\|P_{1}(x) v\right\| .
$$


From $\left(u e g_{3}^{\prime \prime}\right)$ and $\left(u e d_{3}^{\prime}\right)$ we obtain that

$$
e^{\omega t}\left\|C(t, x) P_{3}(x) v\right\| \leq N D e^{|\xi+\omega|}\left\|P_{3}(x) v\right\| .
$$

We shall prove $\left(u e t_{2}^{\prime \prime}\right)$ from Proposition 3.3. If $t \geq 1$, then we obtain that

$$
\begin{gathered}
e^{\nu t}\left\|D_{2}(t, x) P_{2}(S(t, x)) v\right\|=\int_{0}^{1} e^{\nu t}\left\|D_{2}(t, x) P_{2}(S(t, x)) v\right\| d \tau= \\
=\int_{0}^{1} e^{\nu t}\left\|D_{2}(t-\tau+\tau, x) P_{2}(S(t-\tau+\tau, x)) v\right\| d \tau= \\
=\int_{0}^{1} e^{\nu t}\left\|D_{2}(\tau, x) D_{2}(t-\tau, S(\tau, x)) P_{2}(S(t, x)) v\right\| d \tau= \\
=\int_{0}^{1} e^{\nu t}\left\|D_{2}(\tau, x) P_{2}(S(\tau, x)) D_{2}(t-\tau, S(\tau, x)) P_{2}(S(t, x)) v\right\| d \tau \leq \\
\leq \int_{0}^{1} e^{\nu t} N e^{\theta \tau}\left\|P_{2}(S(\tau, x)) D_{2}(t-\tau, S(\tau, x)) P_{2}(S(t, x)) v\right\| d \tau= \\
=N \int_{0}^{1} e^{\nu(t-\tau)} e^{(\theta+\nu) \tau}\left\|D_{2}(t-\tau, S(\tau, x)) P_{2}(S(t, x)) v\right\| d \tau \leq \\
\leq N e^{|\theta+\nu|} \int_{0}^{1} e^{\nu(t-\tau)}\left\|D_{2}(t-\tau, S(\tau, x)) P_{2}(S(t, x)) v\right\| d \tau= \\
=N D e^{|\theta+\nu|}\left\|P_{2}(S(t, x)) v\right\| .
\end{gathered}
$$

For $t \in[0,1)$, we observe that

$e^{\nu t}\left\|D_{2}(t, x) P_{2}(S(t, x)) v\right\| \leq e^{\nu t} N e^{\theta t}\left\|P_{2}(S(t, x)) v\right\|=N D e^{|\theta+\nu|}\left\|P_{2}(S(t, x)) v\right\|$.

We shall prove $\left(u e t_{4}^{\prime \prime}\right)$ from Proposition 3.3. If $t \geq 1$, then we have

$$
\left\|D_{3}(t, x) P_{3}(S(t, x)) v\right\|=\int_{0}^{1}\left\|D_{3}(t, x) P_{3}(S(t, x)) v\right\| d \tau=
$$




$$
\begin{gathered}
=\int_{0}^{1}\left\|D_{3}(\tau, x) D_{3}(t-\tau, S(\tau, x)) P_{3}(S(t, x)) v\right\| d \tau= \\
=\int_{0}^{1}\left\|D_{3}(\tau, x) P_{3}(S(\tau, x)) D_{3}(t-\tau, S(\tau, x)) P_{3}(S(t, x)) v\right\| d \tau \leq \\
\leq \int_{0}^{1} N e^{\xi \tau}\left\|P_{3}(S(\tau, x)) D_{3}(t-\tau, S(\tau, x)) P_{3}(S(t, x)) v\right\| d \tau= \\
=N e^{\eta t} \int_{0}^{1} e^{\eta(\tau-t)} e^{(\xi-\eta) \tau}\left\|D_{3}(t-\tau, S(\tau, x)) P_{3}(S(t, x)) v\right\| d \tau \leq \\
\leq N e^{\eta t} e^{|\xi-\eta|} \int_{0}^{t} e^{\eta(\tau-t)}\left\|D_{3}(t-\tau, S(\tau, x)) P_{3}(S(t, x)) v\right\| d \tau \leq \\
\leq N e^{\eta t} e^{|\xi-\eta|} D\left\|P_{3}(S(t, x)) v\right\| .
\end{gathered}
$$

For $t \in[0,1)$, we observe that

$$
\begin{aligned}
\left\|D_{3}(t, x) P_{3}(S(t, x)) v\right\| & \leq N e^{\xi t}\left\|P_{3}(S(t, x)) v\right\|=N e^{\eta t} e^{(\xi-\eta) t}\left\|P_{3}(S(t, x)) v\right\| \leq \\
& \leq N e^{\eta t} e^{|\xi-\eta|} D\left\|P_{3}(S(t, x)) v\right\| .
\end{aligned}
$$

From Proposition 3.3, we obtain that $(C, \mathcal{P})$ has a uniform exponential trisplitting.

\section{References}

[1] B. Aulbach, J. Kalkbrenner, Exponential forward splitting for noninvertible difference equation, Comput. Math. Appl. 42 (2001), 743-754.

[2] L. Barreira, C. Valls, Noninvertible cocycles: robustness and exponential dichotomies, Discrete and Continuous Dynamical Systems 32 (2012), 4111-4131.

[3] L.E. Biriş, T. Ceauşu, C.L. Mihiţ, On uniform exponential splitting of variational nonautonomous difference equations in Banach spaces, Recent Progress in Difference Equations, Discrete Dynamical Systems and Applications, submitted. 
[4] L.E. Biriş, T. Ceauşu, C.L. Mihiţ, I.-L. Popa, Uniform Exponential Trisplitting - A New Criterion for Discrete Skew-Product Semiflows, Electron. J. Qual. Theory Differ. Equ. 2019, No. 70 (2019), 1-22.

[5] L. Biriş, M. Megan, On a concept of exponential dichotomy for cocycles of linear operators in Banach spaces, Bull. Math. Soc. Sci. Math. Roumanie, 59 (107), No. 3 (2016), 217-223.

[6] L.E. Biriş, R. Retezan, On exponential trichotomy of cocycles over semiflows, An. Univ. Vest Timiş. Ser. Mat.-Inform., LII, 1 (2014), 1727 .

[7] C. Chicone, Y. Latushkin, Evolution Semigroups in Dynamical Systems and Differential Equations, Mathematical surveys and monographs Vol. 70, Amer. Math. Soc., 1999.

[8] S.N. Chow, H. Leiva, Two definitions of exponential dichotomy for skew-product semiflows in Banach spaces, Proc. Amer. Math. Sc. 124 (1996), 1071-1081.

[9] P.E. Kloeden, M. Rasmusen, Nonautonomous Dynamical Systems, American Mathematical Society, Mathematical surveys and monographs Vol. 176, Amer. Math. Soc., 2011.

[10] N.T. Huy, Existence and robustness of exponential dichotomy of linear skew-product semiflows over semiflows, J. Math. Anal. Appl. 333 (2007), 731-752.

[11] Y. Latushkin, S. Montgomery-Smith, T. Randolph, Evolutionary semigroups and dichotomy of linear skew-product flows on locally compact spaces with Banach fibers, J. Differential Equations 125 (1996), 73-116.

[12] Y. Latushkin, R. Schnaubelt, Evolution semigroups, translation algebras and exponential dichotomy of cocycles, J. Differential Equations 159 (1999), 321-369.

[13] M. Megan, I.-L. Popa, Exponential splitting for nonautonomous linear discrete-time systems in Banach spaces, J. Comput. Appl. Math. 312 (2017), 181-191.

[14] C. Pötzsche, Geometric Theory of Discrete Nonautonomous Dynamical Systems, Springer, 2010. 
[15] M. Megan, B. Sasu, A.L. Sasu, Theorems of Perron type for uniform exponential dichotomy of linear skew-product semiflows, Bull. Belg. Math. Soc. Simon Stevin 10 (2003), 143-154.

[16] M. Megan, C. Stoica, L. Buliga, Trichotomy for linear skew-product semiflows, in: Applied Analysis and Differential Equations, World. Sci. Publ. Hackensak, N.J., 2007, 227-236.

[17] M. Megan, C. Stoica, L. Buliga, On asymptotic behaviour for linear skew-evolution semiflows in Banach spaces, Carpathian Journal of Mathematics, 23, No. 1-2 (2007), 117-125.

[18] M. Megan, C. Stoica, Concepts of dichotomy for skew-evolution semiflows on Banach spaces, Annals of the Academy of the Romanian Scientists, Series on Mathematics and Applications 2 (2010), 125-140.

[19] C.L. Mihity, C.S. Stoica, M. Megan, On uniform exponential splitting for noninvertible evolution operators in Banach spaces, An. Univ. Vest Timiş. Ser. Mat.-Inform. LIII, 2 (2015), 121-131.

[20] R.J. Sacker, G.R. Sell, Existence of dichotomies and invariant splittings for linear differential systems I, J. Differential Equations 15 (1974), 429-458.

[21] R.J. Sacker, G.R. Sell, Dichotomies for linear evolutionary equations in Banach spaces, J. Differential Equations 113 (1994), 17-67.

[22] A.L. Sasu, B. Sasu, Admissibility and exponential trichotomy of dynamical systems described by skew-product flows, J. Differential Equations 260 (2016), 1656-1689.

\section{Larisa Elena Biriş}

Department of Mathematics

West University of Timişoara

V. Pârvan Blvd., No. 4

300223 Timişoara

Romania

E-mail: larisa.biris@e-uvt.ro

Claudia Luminiţa Mihiț 
Vol. LVI (2018) Uniform exponential trisplitting

Department of Mathematics

West University of Timişoara

V. Pârvan Blvd., No. 4

300223 Timişoara

Romania

E-mail: mihit.claudia@yahoo.com

Traian Ceauşu

Department of Mathematics

West University of Timişoara

V. Pârvan Blvd., No. 4

300223 Timişoara

Romania

E-mail: traian.ceausu@e-uvt.ro

Ioan-Lucian Popa

Department of Exact Sciences and Engineering

University "1 Decembrie" 1918 of Alba Iulia

510009 Alba Iulia

Romania

E-mail: lucian.popa@uab.ro 